\title{
Efeito da Inserçãa de Implante Anticoncepcional Contendo Acetato de Nomegestrol sobre a Função Ovariana, Muco Cervical e Penetração Espermática
}

\author{
Effects of a Contraceptive Implant Containing Nomegestrol Acetate on \\ Ovarian Function, Cervical Mucus and Sperm Penetration \\ Ione Cristina Barbosa, Elsimar Coutinho, Bruno Matias, \\ Renata Lopes, Antônio Carlos Vieira Lopes
}

\begin{abstract}
RESUM0
Objetivos: estudar o efeito de um único implante de acetato de nomegestrol (Uniplant) sobre função ovariana, produção do muco cervical e penetração espermática, quando inserido na fase pré-ovulatória.

Métodos: estudo clínico aberto, comparativo, incluindo 20 mulheres com ciclos menstruais regulares que foram estudadas durante um ciclo menstrual antes (controle) e um ciclo menstrual depois da inserção do implante. Dosagens de hormônio luteinizante (LH), estradiol e progesterona, ultra-sonografia vaginal, coleta de muco cervical e teste de penetração espermática foram realizados. Para comparação estatística, foi utilizado o Student t-test para grupos pareados e o teste Wilcoxon não paramétrico. Os valores estão mostrados como médias \pm erro padrão. Resultados: todos os ciclos controles foram ovulatórios com parâmetros normais. Os níveis pré-ovulatórios de estradiol e LH diminuíram significativamente de 603,2 $\pm 78,0 \mathrm{pmol} / \mathrm{l}$ e 22,5 \pm 6,5 UI/l na pré-inserção do implante para 380,7 $\pm 51,9 \mathrm{pmol} / \mathrm{l}$ e 4,9 $\pm 1,3 \mathrm{UI} / \mathrm{l} 48$ horas após a inserção ( $p<0,05$ e $p<0,01$, respectivamente). Os níveis de progesterona mantiveram-se sem variação significativa após a inserção do implante (ciclo controle $=49,8 \pm$ $3,3 \mathrm{nmol} / \mathrm{l}$ e ciclo tratado $=43,2 \pm 5,2 \mathrm{nmol} / \mathrm{l})$. Muco cervical e penetração espermática foram profundamente afetados em 10,5\% das usuárias após 20 horas da inserção do implante, em 68,5\% das usuárias após 24 horas e em 100\% após 48 horas. Ruptura folicular ocorreu na maioria dos ciclos tratados após 48 horas da inserção.

Conclusões: o uso de um único implante de acetato de nomegestrol inserido na fase préovulatória afetou os picos pré-ovulatórios de estradiol e $\mathrm{LH}$, a produção do muco cervical e a penetração espermática, mas não foi capaz de impedir a ovulação, o que reforça a necessidade da inserção do implante nos 5 primeiros dias do ciclo menstrual.
\end{abstract}

PALAVRAS-CHAVE: Implante contraceptivo. Acetato de nomegestrol. Uniplant. Muco cervical. Penetração espermática.

\section{Introdução}

O implante subdérmico único de acetato de nomegestrol (Uniplant) libera dose baixa de

Maternidade Climério de Oliveira, Universidade Federal da Bahia

Correspondência:

Ione C. Barbosa

Maternidade Climério de Oliveira

Rua do Limoeiro, no 1

40.055-150 - Salvador - BA

Tel/Fax. (71) 242-4014

Este estudo foi financiado pela Fundação Rockefeller, dentro do programa South-to-South. hormônio e foi demonstrado em vários estudos clínicos como sendo seguro e apresentando mínimos efeitos metabólicos em mulheres saudáveis ${ }^{1}$. Os mecanismos da sua ação contraceptiva, como a de outros implantes progestínicos, podem ser explicados pela ligação a receptores localizados em diversas células-alvo, distribuídas no eixo hipotalâmico-pituitário-gonadal-trato genital. Implantes progestínicos interferem nos processos requeridos para o encontro de gametas e a fertilização. Deste modo, interferem principalmente com o processo ovulatório, inibindo parcial ou completamente o pico gonadotrófico e modificando a qualidade do muco cervical (MC) ${ }^{2}$. 
Foi demonstrado que a liberação de levonorgestrel por um sistema contraceptivo intrauterino exerce efeito na função ovariana que consiste em distúrbios do crescimento e da rotura folicular e níveis plasmáticos mais baixos de $\mathrm{E}_{2}$, LH e $\mathrm{P}^{3,4}$. Os autores também constataram que, em mulheres com ciclos menstruais regulares, $61,1 \%$ dos ciclos tiveram MC considerado pobre, de acordo com os critérios da Organização Mundial de Saúde ${ }^{5}$.

Estudos com implante de acetato de nomegestrol ${ }^{6}$ demonstraram que a produção do MC é afetada durante dois anos consecutivos de uso. Nesses estudos, o implante foi inserido entre o primeiro e o quinto dia do ciclo menstrual e a coleta do MC iniciada no oitavo dia do ciclo menstrual. Era desconhecido o exato momento em que a produção do $\mathrm{MC}$ e a penetração de espermatozóides sofrem alterações após a inserção do implante. Um estudo prévio $^{7}$ demonstrou que o implante de acetato de nomegestrol não teve nenhum efeito sobre a produção do MC nas primeiras 12 horas depois de sua inserção. O objetivo do presente estudo foi verificar, em um número maior de usuárias, o tempo requerido por este implante para exercer seus efeitos sobre a função ovariana, produção de MC e sobre o teste de penetração de espermatozóides, em um momento em que o processo de crescimento folicular e produção do MC já haviam sido iniciados.

\section{Pacientes e Métodos}

Mulheres em idade fértil, solicitando método anticoncepcional hormonal, foram convidadas a participar deste estudo, previamente aprovado pelo Comitê de Ética em Pesquisa da Maternidade Climério de Oliveira. Todas as mulheres incluídas no estudo assinaram o Termo de Consentimento Livre e Esclarecido. Nenhum tipo de conflito de interesse existiu que pudesse comprometer o desenvolvimento e/ou conclusões deste trabalho.

Vinte voluntárias saudáveis, com idade média de $25,3 \pm 0,8$ anos, média de peso de $55,2 \pm 1,8$ $\mathrm{kg}$, média da altura de $164,0 \pm 2,1 \mathrm{~cm}$, foram incluidas neste estudo. Todas tinham história de ciclos menstruais regulares, com média de 27,9 $\pm 0,3$ dias. Todas tiveram gestações (média de 1,8 $\pm 0,3$ vezes) e tinham parido em média $1,2 \pm 0,1$ crianças. Não tinham usado contraceptivo hormonal ou DIUs por período mínimo de seis meses antes do início do estudo. Os casais usaram preservativos para contracepção durante os dois ciclos do estudo. Durante o período da coleta do MC, solicitou-se aos casais não manterem relações sexuais, mesmo com o uso de preservativos. Também se solici- tou que as mulheres não usassem nenhum inibidor de sintese de prostaglandinas por pelo menos três meses antes do estudo e durante o período do estudo. Todas as pacientes foram avaliadas durante um ciclo antes da inserção do implante e durante o ciclo no qual o implante foi inserido. O implante de acetato de nomegestrol (Uniplant, South-to-South Cooperation in Reproductive Health, Brazil) é um novo contraceptivo de longa duração derivado da 19norprogesterona.

Os implantes foram manufaturados com tubos de dimetilpolissiloxano fabricados para uso médico (silástico, número de catálogo 602-265, da Dow Corning; Midland, Michigan) e confeccionados com segmentos de tubo medindo 39 milímetros de comprimento total (35 milímetros preenchidos) e 2,4 milímetros de diâmetro. Os tubos de silástico foram preenchidos com $55 \mathrm{mg}( \pm 10 \%)$ de acetato de nomegestrol cristalino e finamente pulverizado (3,20-oxo-6-metil-17- $\alpha$-acetoxi-19norpregna-4,6-dieno; Theramex, França) e selados em ambas as extremidades com adesivo de silástico de padrão médico tipo A. Os implantes foram esterilizados em autoclave. Após anestesia subcutânea com procaína a $2 \%$, realizou-se para cada participante a inserção subcutânea de um único implante na região glútea, conforme descrito previamente ${ }^{8}$.

Os implantes foram inseridos em todas as voluntárias quando o escore do MC alcançou 8-10, baseados nos seguintes parâmetros: volume, consistência, cristalização, filância e celularidade. Os escores de MC, variando de 0-3 para cada parâmetro foram calculados da seguinte forma: volume: $0=0 \mathrm{~mL}, 1=0,1 \mathrm{~mL}, 2=0,2 \mathrm{~mL}, 3 \geq 0,3$ $\mathrm{mL}$; consistência: $0=$ espesso/altamente viscoso, 1 = tipo intermediário/viscoso, 2 = moderadamente viscoso, 3 = muco normal para o meio do ciclo (pré-ovulatório); cristalização: $0=$ nenhuma cristalização, 1 = cristalização atípica, $2=$ cristalização primária e secundária, $3=$ cristalização terciária e quaternária; filância: $0<1 \mathrm{~cm}, 1=1-4$ $\mathrm{cm}, 2=5-8 \mathrm{~cm}, 3 \geq 9 \mathrm{~cm}$; celularidade: $0 \geq 11$ células $/ \mathrm{HPF}(40 \times 10), 1=6-10,2=1-5,3=0$ células. As inserções foram realizadas independentes do dia do ciclo menstrual. Em sete das 20 mulheres as inserções ocorreram quando o escore do $\mathrm{MC}$ foi 10 , em onze mulheres quando o escore do MC foi 9 e em duas mulheres quando este foi 8 . Em uma mulher o implante foi inserido no $10^{\circ}$ dia do ciclo. Em quatro mulheres a inserção foi feita no $11^{\circ}$ dia, três no $12^{\circ}$ dia, nove no $13^{\circ}$ dia, duas no $15^{\circ}$ dia e em uma, no $16^{\circ}$ dia do ciclo menstrual.

A escolha do dia para a inserção foi baseada somente no escore do MC. As amostras de sangue venoso foram colhidas em dias alternados a partir do $8^{\circ}$ dia do ciclo, até que houvesse evidência ultra- 
sonográfica de um folículo medindo $10,0 \mathrm{~mm}$ e o escore do MC fosse maior do que 5; a partir de então, diariamente, até a evidência ultrasonográfica de ruptura folicular. Em seguida, as amostras foram colhidas em dias alternados, até o próximo sangramento menstrual.

Para a coleta do muco, um tubo fino de polietileno foi adaptado a uma seringa de $10 \mathrm{~mL}$ e introduzido no orifício externo nos mesmos dias em que as amostras de sangue venoso foram coletadas. O escore do MC foi determinado de acordo com os critérios da Organização Mundial de Saúde $(\mathrm{OMS})^{5}$. O escore máximo para o MC é 15. Um escore superior a 10 é indicativo de $\mathrm{MC}$ satisfatório, favorecendo a penetração de espermatozóides, ao passo que um escore menor do que 10 representa MC desfavorável. O MC foi avaliado imediatamente após a coleta. O muco foi colocado em lâmina e deixado secar. Após 2 horas, quatro campos microscópicos (HPF; 40 x 10) foram examinados a fim de se estudar a cristalização e a celularidade. O MC foi coletado $20,32,48,60,72$, 84 e 96 horas após a inserção.

O MC foi colocado em um tubo capilar. Uma extremidade do tubo foi selada com massa de modelar. O MC foi mantido a $-20^{\circ} \mathrm{C}$, por período máximo de quatro meses, até o teste de penetração de espermatozóides (TPE) ser analisado. A penetração de espermatozóides no MC foi avaliada de acordo com os critérios da OMS ${ }^{5}$. Depois que todas as amostras do muco foram descongeladas, foram feitos os TPEs com a mesma amostra previamente analisada, encontrando-se dentro dos limites normais para volume, densidade, morfologia e migração. A extremidade aberta do tubo capilar foi colocada em reservatório contendo a amostra do sêmen, em uma incubadora a gás com temperatura de $37^{\circ} \mathrm{C}$, por uma hora. O escore foi calculado baseado na penetração linear, na densidade da penetração e na motilidade, de acordo com as seguintes definições:

Penetração linear: a profundidade da penetração = a maior distância $(\mathrm{em} \mathrm{cm})$ alcançada pelos espermatozóides mais avançados. Densidade da penetração: o número de espermatozóides em áreas selecionadas do tubo capilar (LPF 10 x 10). Motilidade: a motilidade para adiante dos espermatozóides no terço superior do tubo capilar foi analisada como segue: $0=$ sem progressão; $1=$ 25\% dos espermatozóides têm motilidade progressiva; 2 = 25-50\% dos espermatozóides têm motilidade progressiva; $3=>50 \%$ dos espermatozóides têm motilidade progressiva.

Escore máximo de 9 representa um excelente teste. O escore minimo de 0 representa teste negativo. Entre esses valores, escore de 3 representa um teste deficiente, ao passo que 6 representa teste satisfatório (teste de Kramer).
O padrão de crescimento folicular foi avaliado em todas as voluntárias por ultra-sonografia transvaginal, fazendo-se a varredura de ambos os ovários e usando-se a técnica descrita por Osmers ${ }^{9}$ e Goswamy ${ }^{10}$. Antes da realização do exame é solicitado que a paciente esvazie a bexiga urinária para eliminar qualquer risco de que uma bexiga distendida possa interferir com a forma da cavidade uterina e a qualidade do exame ultrasonográfico. A técnica consiste em localizar os ovários por meio de varredura no eixo látero-lateral (transverso), deslocando o transdutor do fundo uterino em direção ao lado do ovário a ser examinado, tentando seguir os prolongamentos dos anexos uterinos aí inseridos, como a trompa, o ligamento útero-ovárico e o mesossalpinge, que aparecem como imagem hipoecóica fina e alongada. Caso o ovário não seja encontrado, deve-se bascular o transdutor no sentido ântero-posterior e vice-versa, de forma delicada, sendo que em algumas pacientes obesas ou com interposição de alças intestinais com gases, pode-se aplicar a compressão anterior do baixo ventre com a mão livre. Se mesmo assim ele não for localizado, a varredura deve passar para o eixo longitudinal, basculando o transdutor da linha mediana para o lado, até encontrar os vasos ilíacos internos, onde habitualmente os ovários estão localizados. Estes encontrados, passamos à fase de varredura completa da gônada, tanto no sentido longitudinal como no transverso, avaliando a sua forma, superfície, contornos, relação com o útero e as estruturas vizinhas, também avaliando a ecotextura do parênquima e achados como folículos, corpo lúteo, cistos e calcificações, devendo detalhá-los e medilos. O último passo é a medida do volume ovariano, dividindo o visor em duas imagens, sendo uma no maior eixo (longitudinal) e a outra no menor (transverso), coletando-se os diâmetros longitudinais e ântero-posterior no primeiro e o transversal no segundo. O volume é calculado multiplicando estes valores entre si e depois pela constante 0,52 (L x $\mathrm{AP}$ x T x 0,52). Para finalizar, deve-se registrar estas imagens e suas respectivas medidas em fotos.

$\mathrm{O}$ exame começou no $8^{\circ}$ dia do ciclo e foi repetido em dias alternados, até a evidência ultrasonográfica de um folículo de $10 \mathrm{~mm}$; a partir de então, diariamente até ocorrer evidência ultrasonográfica de ruptura folicular e, depois disso, em dias alternados até o início do sangramento menstrual seguinte. A espessura e a textura endometriais foram medidas da interface ecogênica proximal à distal na junção entre o endométrio e o miométrio, por calibrador eletrônico interno embutido no equipamento. Somente a seção longitudinal do útero foi usada para a medida da espessura uterina e endometrial. A avaliação da espessura e textura endometrial foi feita de acor- 
do com os estudos prévios ${ }^{11,12}$. O eco linear central representa a cavidade endometrial, freqüentemente circundada por dois ecos lineares, representando a função mioendometrial. As camadas hipoecogênicas que cercam o eco linear central representam o crescimento endometrial. O crescimento da ecogenicidade, a partir das bordas dos ecos mioendometriais, representam mudanças diferenciais das camadas endometriais.

A progesterona foi dosada por radioimunoensaio usando-se kits comerciais da Diagnostic Products Corporation, Los Angeles, CA, USA. A sensibilidade foi de 0,09 $\mathrm{nmol} / \mathrm{L}$ e o coeficiente de variação entre ensaios de $6,4 \%$. Os valores normais estavam entre 0,3-89,0 nmol/L. O estradiol foi determinado por radioimunoensaio, usando-se kits comerciais da Diagnostic Products Corporation. A sensibilidade foi de 5,3 pmol/L e o coeficiente de variação entre ensaios, de $5,5 \%$. Os valores normais estavam entre $146,0-1468,0 \mathrm{pmol} / \mathrm{L}$. O LH foi determinado por radioimunoensaio, usando-se kits comerciais da Diagnostic Products Corporation. A sensibilidade foi de 1,21 UI/L e o coeficiente de variação entre ensaios, de $8,3 \%$. Os valores normais estavam entre 15-90 UI/L.

Para comparação estatística, foi utilizado o teste $t$ de Student para grupos pareados e o teste Wilcoxon não paramétrico. Os valores estão mostrados como médias \pm erro padrão da média.

\section{Resultados}

A média do escore do MC pré-ovulatório para os ciclos controle foi $14,6 \pm 0,2$. No ciclo tratado, o implante de acetato de nomegestrol foi inserido quando a média do escore do MC foi $10,3 \pm 0,1$. Vinte horas após a inserção do implante, a média do escore do MC foi de 7,4 $\pm 0,5,24$ horas após a inserção foi de $6,5 \pm 0,3$ e após 48 horas a média do escore do MC foi 3,1 $\pm 0,2$ (Tabela 1).

Tabela 1 - Escore do muco cervical nos ciclos controle e ciclos tratados antes e depois da inserção do implante.

\begin{tabular}{lc}
\hline Ciclos controle & Escore do muco cervical \\
\hline Antes da inserção do implante & $14,6 \pm 0,2(n=20)$ \\
20 h após inserção do implante & $10,3 \pm 0,1 \quad(n=20)$ \\
24 h após inserção do implante & $7,4 \pm 0,5^{*}(n=20)$ \\
48 h após inserção do implante & $6,5 \pm 0,3^{*}(n=20)$ \\
${ }^{*} p<0,01$ & $3,1 \pm 0,2^{*}(n=20)$ \\
Todos os níveis: média \pm EPM.
\end{tabular}

A média da espessura endometrial no ciclo tratado foi de $12,5 \pm 0,3 \mathrm{~mm}$. Não se observou ne- nhuma diferença estatisticamente significante quando os ciclos tratados foram comparados aos ciclos controle.

Para os ciclos controle, a média do escore para o teste de penetração de espermatozóides foi de $7,6 \pm 0,3$. A média do escore para o teste de penetração do espermatozóide no ciclo tratado, an-

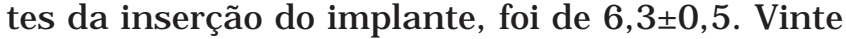
horas após a inserção do implante, a média do escore foi de 4,3 $\pm 0,3,24$ horas após foi de 3,6 $\pm 0,5$ e 48 horas após a inserção do implante, a média do escore para o teste de penetração do espermatozóide foi de $2,1 \pm 0,5$. Essas diferenças foram estatisticamente significantes quando comparadas à média do escore antes da inserção do implante, assim como à do grupo controle.

Todas as mulheres tiveram ciclos controle ovulatórios, com desenvolvimento, ruptura folicular, produção de MC, teste de penetração de espermatozóides e níveis hormonais dentro dos limites normais. Nos ciclos tratados, das vinte mulheres estudadas, duas ovularam 20 horas após a inserção do implante, quatro mulheres após 24 horas, onze após 48 horas, uma após 72 horas, uma após 84 horas e uma após 96 horas da inserção do implante.

A média dos níveis plasmáticos de estradiol 48 horas antes da ovulação e da inserção do implante foi de $603,2 \pm 78,0 \mathrm{pmol} / \mathrm{L}$, diminuindo para $492,6 \pm 86,0$ em 24 horas e $380,7 \pm 51$, 9 em 48 horas após a inserção do implante. A média do pico máximo de estradiol plasmático, no ciclo controle, foi de 986,5 $\pm 87,3 \mathrm{pmol} / \mathrm{L}$. Esta diferença foi estatisticamente significativa $(\mathrm{p}=0,0025)$ quando comparada ao ciclo em que o implante foi inserido (Tabelas 2 e 3).

Tabela 2 - Níveis hormonais nos ciclos controle e tratados.

\begin{tabular}{lcc}
\hline & Ciclos controle & Ciclos tratados \\
\hline Pico de estradiol pré-ovulatório (pmol/L) & $986,5 \pm 87,3$ & $603,2 \pm 78,0^{*}$ \\
Pico de LH pré-ovulatório (UI/L) & $38,2 \pm 4,3$ & $10,2 \pm 2,6^{*}$ \\
Pico de progesterona luteal (nmol/L) & $49,8 \pm 3,3$ & $43,2 \pm 5,2$ \\
Pico de estradiol luteal (pmol/L) & $679,5 \pm 47,3$ & $709,6 \pm 98,9$ \\
\hline${ }^{*} p<0,01$ & & \\
Todos os níveis: média \pm EPM. & &
\end{tabular}

A média dos níveis do LH plasmático 48 horas antes da ovulação e pré-inserção do implante

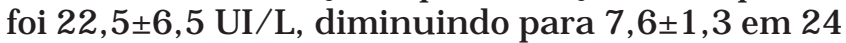

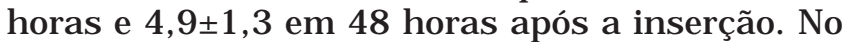
ciclo controle, a média do pico pré-ovulatório de $\mathrm{LH}$ foi $38,2 \pm 4,3 \mathrm{UI} / \mathrm{L}$. Esta diferença foi estatisticamente significativa $(\mathrm{p}=0,0045)$, comparada ao ciclo em que o implante foi inserido (Tabela 3). 
Tabela 3 - Níveis hormonais e diâmetro folicular pré e pós-inserção do implante.

\begin{tabular}{lccc}
\hline & $\begin{array}{c}\text { Níveis de } \\
\text { LH (UI/L) }\end{array}$ & $\begin{array}{c}\text { Níveis de } \\
\text { estradiol (pmol/L) }\end{array}$ & $\begin{array}{c}\text { Diâmetro } \\
\text { folicular (mm) }\end{array}$ \\
\hline Pré-inserção do & $22,5 \pm 6,5$ & $603,2 \pm 78,0$ & $19,2 \pm 0,2$ \\
$\quad$ implante & $(n=20)$ & $(n=20)$ & $(n=20)$ \\
24 h após inserção do & $7,6 \pm 1,3^{*}$ & $492,6 \pm 86,0$ & $18,7 \pm 0,5$ \\
$\quad$ implante & $(n=20)$ & $(n=20)$ & $(n=20)$ \\
48 h após inserção do & $4,9 \pm 1,3^{* *}$ & $380,7 \pm 51,9^{*}$ & $18,6 \pm 0,7$ \\
implante & $(n=20)$ & $(n=20)$ & $(n=20)$ \\
\hline${ }^{*} p<0,05$ & & & \\
${ }^{* *} p<0,01$ & & & \\
Todos 0s níveis: média \pm EPM & &
\end{tabular}

Não se observou diferença significativa $(\mathrm{p}=0,055)$ nos níveis plasmáticos de progesterona quando comparados aos níveis do ciclo controle. Os niveis de progesterona foram mais baixos do que os controles, mas esta diferença não foi estatisticamente significante. No ciclo tratado, a média dos níveis plasmáticos de progesterona foi $43,2 \pm 5,2$, ao passo que no ciclo controle foi 49,8 $\pm 3,3 \mathrm{nmol} / \mathrm{L}$ (Tabela 2).

Não se observou diferença estatisticamente significante na média dos níveis do pico luteal de estradiol, comparados aos do ciclo controle (Tabela 2).

A média do diâmetro folicular, 48 horas antes da ovulação e da inserção do implante, foi $19,0 \pm 0,2$. Vinte e quatro horas após a inserção do implante, a média do diâmetro folicular foi $18,7 \pm 0,5$, e 48 horas após a inserção do implante e pré-ovulação, a média do diâmetro folicular foi 18,6 $\pm 0,7$ mm (Tabela 3).

\section{Discussão}

O acetato de nomegestrol é um derivado da 19-norprogesterona, com potente atividade progestacional e nenhum efeito androgênico ${ }^{8}$. A liberação através da membrana de silástico é muito estável em toda a duração de seu uso ${ }^{13}$. Neste estudo, $55 \mathrm{mg}$ de acetato de nomegestrol foram usados em uma cápsula única de silástico.

A secreção do MC humano tem importante papel no transporte, sobrevivência e capacitação dos espermatozóides. O MC muda suas características durante todo o ciclo menstrual,seguindo as mudanças cíclicas nos níveis de estradiol e progesterona. No momento da ovulação, o produto secretado tornase abundante, aquoso e viscoso, e o componente mucinoso é formado como longas fibrilas ${ }^{14-16}$. Fator comum aos progestagênios é a sua capacidade de tornar o MC viscoso, escasso e impenetrável aos espermatozóides ${ }^{2}$. Estudos prévios demonstraram que o implante contendo acetato de nomegestrol tem efeito potente na produção do MC nas mulheres, durante período de 24 meses de uso ${ }^{6}$. Esses achados estão de acordo com estudos anteriores com implantes de levonorgestrel $\left(\right.$ Norplant $\left.^{\circledR}\right)$ e com o sistema intra-uterino de liberação de levonorgestrel $\left(\text { Mirena }{ }^{\circledR}\right)^{6,17-19}$. O potente efeito sobre o muco cervical observado nesse estudo comunga com relatos anteriores de estudos realizados com outros progestínicos de ação prolongada ${ }^{20-22}$. Esse efeito foi também descrito em usuárias de implante que libera etonogestrel (Implanon $\left.{ }^{\circledR}\right)^{23,24}$. Esta ação sob o muco cervical é, portanto, um efeito adicional que contribui para a eficácia dos contraceptivos contendo apenas progestagênio.

O presente estudo confirma que, quando inserido na fase folicular periovulatória, o implante de acetato de nomegestrol pode afetar os picos préovulatórios de LH e estradiol, em todas as mulheres estudadas, não sendo, porém, capaz de inibir a ovulação. Por outro lado, há registro na literatura de que óvulos coletados após picos de LH de magnitude reduzida apresentam menor capacidade de fertilização in vitro ${ }^{25}$. Os níveis de progesterona foram mais baixos que os do ciclo controle, mas essas diferenças não foram estatisticamente significantes.

Discretas alterações no crescimento folicular foram observadas em 12 das 20 mulheres estudadas. Essas alterações consistiram em apenas uma ligeira diminuição no volume folicular. Dezenove dos 20 ciclos foram ovulatórios, de acordo com a ultra-sonografia e os níveis plasmáticos de progesterona. O implante de acetato de nomegestrol afetou a produção do MC e a penetração do espermatozóide 20 horas após a inserção do implante em $10,5 \%$ e após 24 horas em $68,5 \%$ dos ciclos estudados. Quarenta e oito horas após a inserção, Uniplant afetou a produção do MC e o teste de penetração de espermatozóides em 100\% dos ciclos estudados. Esses resultados estão de acordo com os achados em um estudo previamente publicado ${ }^{7}$.

Baseado nos resultados destes estudos e seguindo as orientações da OMS e FEBRASGO, deve-se orientar as usuárias de todos esses métodos citados acima para utilizarem métodos contraceptivos de barreira, por período de no mínimo vinte e um dias, caso o uso desses progestínicos de longa duração seja iniciado após o quinto dia do ciclo menstrual.

Este estudo demonstrou e confirmou que o implante de acetato de nomegestrol (Uniplant) pode interromper o processo de produção do $\mathrm{MC}$ e afetar a penetração de espermatozóides, assim como afetar os picos pré-ovulatórios de LH e de estradiol, mas ele é incapaz de prevenir a ovulação quando inserido na fase periovulatória, o que reforça a necessidade da inserção do implante nos 5 primeiros dias do ciclo menstrual. 


\section{ABSTRACT}

Objective: to study the effect of a single contraceptive implant of nomegestrol acetate (Uniplant) on the ovarian function, cervical mucus production and sperm penetration, when inserted in women in the preovulatory phase.

Methods: twenty women with regular menstrual cycles were included in an open comparative study. All participants were investigated during one menstrual cycle before (control) and one menstrual cycle after implant insertion. Measurements of estradiol, LH, and progesterone, as well as transvaginal sonography, cervical mucus examination and sperm penetration test, were carried out. Statistical analysis was performed with the paired t-test and the non-parametric test of Wilcoxon.

Results: all control cycles were ovulatory and presented normal parameters. Preovulatory estradiol and LH peak decreased significantly from $603.2 \pm 78.0$ pmo/l and $22.5 \pm$ $6.5 \mathrm{IU} / \mathrm{l}$ at pre-insertion to $380.7 \pm 51.9 \mathrm{pmol} / \mathrm{l}$ and $4.9 \pm 1.3$ IU/ 48 hours after implant insertion $(p<0.05$ and $p<0.01$, respectively). Progesterone levels did not vary significantly (control cycle $=49.8 \pm 3.3 \mathrm{nmol} / \mathrm{l}$ and treated cycle $=43.2 \pm$ $5.2 \mathrm{nmol} / \mathrm{l})$. Cervical mucus and sperm penetration tests were profoundly affected in $10.5 \%$ of the users $20 \mathrm{~h}$ after implant insertion, in $68.5 \%$ after $24 \mathrm{~h}$ and in $100 \%$ after $48 \mathrm{~h}$. Follicular rupture occurred in the majority of the cycles $48 \mathrm{~h}$ after implant insertion.

Conclusions: the use of a single implant of nomegestrol acetate affected estradiol and LH preovulatory peaks and disrupted the process of cervical mucus production and sperm penetration, but it was unable to prevent ovulation when inserted at the preovulatory phase, which reinforces the need to insert the implant during the first five days of the menstrual cycle.

KEYWORDS: Contraceptive implant. Nomegestrol acetate. Uniplant. Cervical mucus. Sperm penetration.

\section{Referências}

1. Dorflinger LJ. Metabolic effects of implantable steroids contraceptives for women. Contraception 2002; 65:47-62.

2. Croxatto HB. Mechanisms that explain the contraceptive action of progestin implants for women. Contraception 2002; 65:21-7.

3. Barbosa I, Bakos O, Olsson SE, Odlind V, Johansson ED. Ovarian function during use of a levonorgestrelreleasing IUD. Contraception 1990; 42:51-66.

4. Barbosa I, Olsson SE, Odlind V, Goncalves T, Coutinho E. Ovarian function after seven years' use of a levonorgestrel IUD. Adv Contracept 1995; 11:85-95.

5. Belsey MA, Eliasson R, Gallegos AJ, Moghissi KS, Paulsen CA, Prassad AM, editors. WHO laboratory manual for the examination of human and semen-cervical mucus interaction. $1^{\text {st }}$ ed. Singapore: Press Concern; 1980

6. Barbosa IC, Coutinho EM, Hirsch C, Ladipo O, Olsson SE, Ulmsten U. Effects of a single contraceptive Silastic implant containing nomegestrol acetate on ovarian function and cervical mucus production during 2 years. Fertil Steril 1996; 65:724-9.
7. Barbosa IC, Coutinho E, Hirsch C, Ladipo OA, Olsson SE, Ulmsten U. Temporal relationship between Uniplant insertion and changes in cervical mucus. Contraception 1996; 54:213-7.

8. Coutinho EM. One year contraception with a single subdermal implant containing nomegestrol acetate (Uniplant). Contraception 1993; 47:97-105.

9. Osmers R. Transvaginal sonography in endometrial cancer. Ultrasound Obstet Gynecol 1992; 2:2-3.

10.Goswamy RK. Transvaginal ultrasonography. BMJ 1992; 304:331-2.

11.Sakamoto C. Sonographic criteria of phasic changes in human endometrial tissue. Int $J$ Gynaecol Obstet 1985; 23:7-12.

12.Yoshimitsu K, Nakamura G, Nakano H. Dating sonographic endometrial images in the normal ovulatory cycle. Int J Gynaecol Obstet 1989; 28:33-9.

13.Ezan E, Benech H, Bucourt R, et al. Enzyme immunoassay for nomegestrol acetate in human plasma. $\mathrm{J}$ Steroid Biochem Mol Biol 1993; 46:507-14.

14. Odeblad E. Biophysical techniques of assessing cervical mucus and microstructure of cervical epithelium. In: Elstein M, Moghissi KS, Barth R, editors. Cervical mucus in human reproduction. $1^{\text {st }}$ ed. Copenhagen: Scriptor; 1973. p. 58-74.

15. Chretien FC. La glaire cervicale III. Roles physiologiques. J Gynecol Obstet Biol Reprod 1977; 6:451-88.

16. Chang MC, Hunter RHF. Capacitation of mammalian sperm: biological and experimental aspects. In: Hamilton DW, Greep RO, editors. Endocrinology. $1^{\text {st }}$ ed. Washington: American Physiological Society; 1975. v. 5, p. 339-51. (Handbook on Physiology, 7).

17.Alvarez F, Brache V, Tejada AS, Faundes A. Abnormal endocrine profile among women with confirmed or presumed ovulation during long-term Norplant use. Contraception 1986; 33:111-9.

18.Shaaban MM, Segal S, Salem HT, Ghaneimah SA, Khalifa EA, Ahmed AG. Sonographic assessment of ovarian and endometrial changes during long-term Norplant use and their correlation with hormonal levels. Fertil Steril 1993; 59:998-1002.

19. Shoupe D, Horenstein J, Mishell DR Jr, Lacarra M, Medearis A. Characteristics of ovarian follicular development in Norplant users. Fertil Steril 1991; 55:76670.

20.Moghissi KS, Marks C. Effects of microdose norgestrel on endogenous gonadotropic and steroid hormones, cervical mucus properties, vaginal cytology, and endometrium. Fertil Steril 1971; 22:424-34.

21.Brache V, Faúndes A, Johansson E, Alvarez F. Anovulation, inadequate luteal phase and poor sperm penetration in cervical mucus during prolonged use of Norplant implants. Contraception 1985; 31:261-73.

22. Croxatto HB, Diaz S, Salvatierra AM, Morales P, Ebensperger C, Brandeis A. Treatment with Norplant subdermal implants inhibits sperm penetration through cervical mucus in vitro. Contraception 1987; 36:193-201.

23. Croxatto HB, Mäkäräinen L. The pharmacodynamics and efficacy of Implanon. An overview of the data. Contraception 1998; 58 Suppl:91S-97S.

24.Davies GC, Feng LX, Newton JR, Van Beek A, CoelinghBennink HJ. Release characteristics, ovarian activity and menstrual bleeding pattern with a single contraceptive implant releasing 3-ketodesogestrel. Contraception 1993; 47:251-61.

25.Verpoest WM, Cahill DJ, Harlow CR, Hull MG. Relationship between midcycle luteinizing hormone surge quality and oocyte fertilization. Fertil Steril 2000; 73:75-7.

Recebido em: 30/5/2003 Aceito com modificações em: 28/5/2004 